Discussion Paper 75

Institute for Empirical Macroeconomics

Federal Reserve Bank of Minneapolis

250 Marquette Avenue

Minneapolis, Minnesota 55480-0291

October 1992

\title{
EMPIRICAL CROSS-SECTION DYNAMICS IN ECONOMIC GROWTH
}

\author{
Danny Quah* \\ London School of Economics
}

*I thank the Institute for Empirical Macroeconomics at FRB Minneapolis for its hospitality, and John Geweke, Ed Green, Nobu Kiyotaki, and Ed Prescott for helpful comments.

Any opinions, findings, conclusions, or recommendations expressed herein are those of the author(s) and not necessarily those of the National Science Foundation, the University of Minnesota, the Federal Reserve Bank of Minneapolis, or the Federal Reserve System. 


\section{Introduction}

Economic growth-across countries, regions, states-has recently been the subject of intensive empirical study; see among many others Barro [1991] and Barro and Sala-i-Martin [1991, 1992]. Grossly over-simplifying, the aims of this work are two-fold: (a) to examine the determinants of long-run growth and (b) to check if, after conditioning on the hypothesized explanatory variables given under (a), income per economic agent converges towards a steady state growth path, possibly differing across economies. ${ }^{1}$ The standard approach in these studies is the following:

(i) calculate the average growth rate over time for each economy;

(ii) across economies regress that average growth rate computed in (i) on pointin-time or time-averaged conditioning variables-schooling, investment, government spending, political circumstances-and an initial income level.

The idea underlying this procedure is that the conditioning variables explain the permanent growth component or trend, while the initial condition controls for transitory dynamics. A very clear discussion of this appears on pp. 224-227 of Barro and Sala-i-Martin [1992]. The results from this analysis have been rich and provocative: ensuing insights range from verifying the neoclassical growth model's predictions to suggesting new directions for research in endogenous growth theory.

Implicit in this empirical work, however, is a view that every economy has a steady-state growth path, well-approximated by a time trend. Such a view is necessary for the time-averaged" growth rate-the left-hand side in these crosscountry regressions-to measure anything sensible, and thus for its covariation with proposed explanatory variables to indicate something structural.

The first part of this paper examines if the cross-country income data bear out such an implicit assumption: the answer turns out to be no. The second part of the paper then turns to an alternative econometric strategy designed specifically to analyze the dynamics in the rich cross-section of country incomes. Conclusions and potential extensions are given in the last section.

\footnotetext{
1 Hereafter I use economy to refer to either countries, regions, or states.
} 


\section{Stable Growth Paths?}

A defining feature of all empirical cross-country growth studies is the interest in how an inherently dynamic phenomena-long- or medium-run growth-varies across different economies in a cross-section. The time dimension of the data for each cross-sectional unit need not be very large, and in particular, typically has only the same order of magnitude as the total number of cross-sectional units under study. The standard approach, described above, collapses dynamic characteristics into a single summary statistic-an average or trend growth rate-and then asks how this statistic covaries in the cross-section with proposed explanatory factors. Such an approach is informative if permanent movements in income were well-described by smooth time trends, themselves largely unaffected by ongoing economic disturbances; or, if significant, large economic shocks occurred only at the beginning of the sample, and thus the smooth time-trends approximation is good, regardless of the true underlying structure.

Unfortunately, neither of these scenarios appears to well-describe the crosscountry income data. Figure 1 shows results from fitting linear time trends, country by country, to the log of per capita income for 118 countries. ${ }^{2}$ It graphs the slope of each economy's time trend after $1973 / 4$ against that before. In practically all cases OLS and heteroskedasticity-robust standard errors show the change to be significant. Of the 118 economies on this graph, 92 (or 78\%) are below the 45 degree line through the origin-a considerable fraction of these, far below.

Figure 2 similarly graphs the standard deviation of $(\log )$ income fluctuations about the fitted (broken) trend line after 1973/4 against the same measure, before. Of the 118 economies, 85 have datapoints above the 45 degree line, indicating $72 \%$ of these economies experienced an increase--some, substantial-in income variability. Imposing a smooth trend, i.e., not allowing a break, as done in traditional strategies only magnifies this increase. ${ }^{3}$

2 The time sample is annual, 1962 through 1985; the regressions are estimated by OLS, with a change permitted in the coefficients on constant and time trend at $1973 / 4$. The data source and a list of the different economies studied here are given in the Data Appendix.

3 Figures 1 and 2 can be viewed as informal ways of examining nonstationarities 
Figure 1

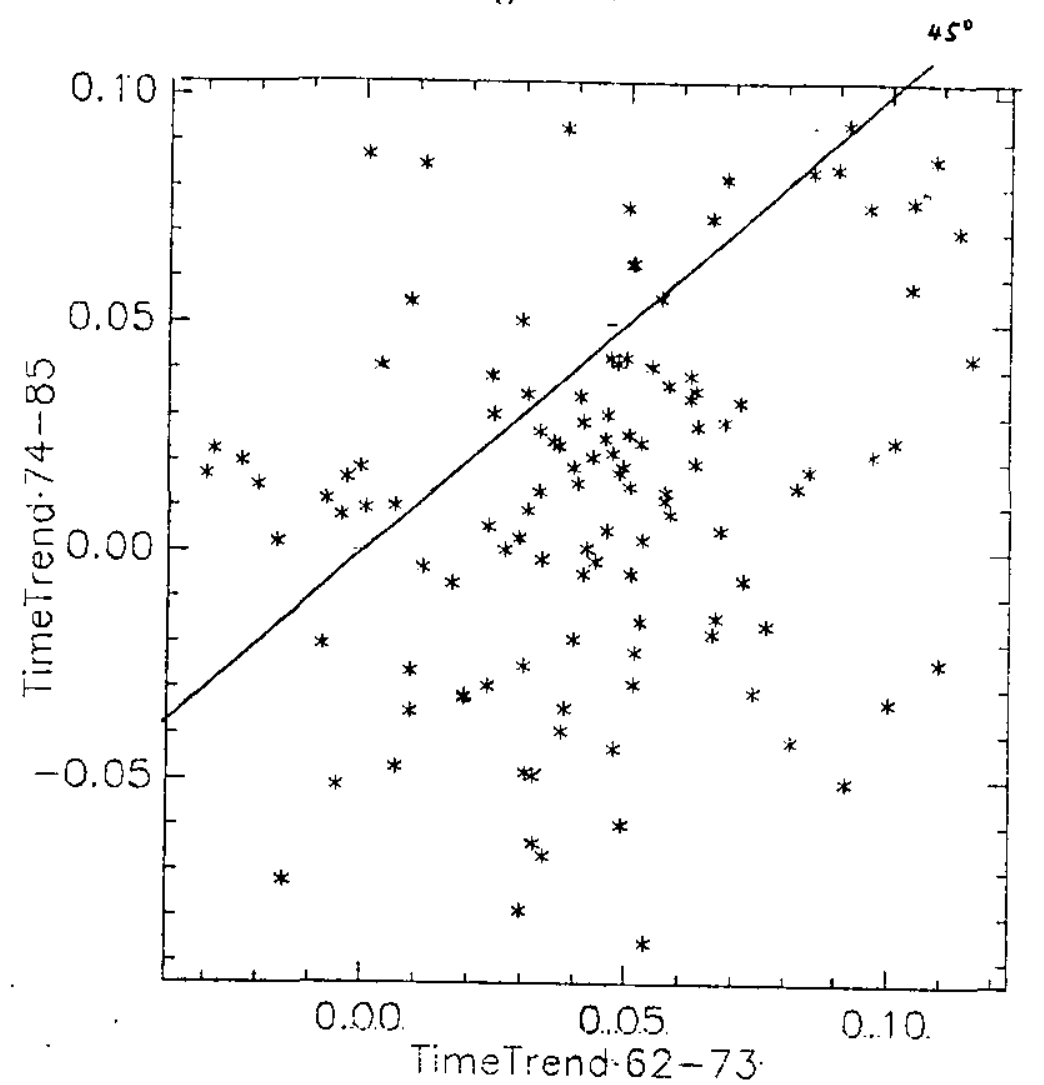

Figure 2

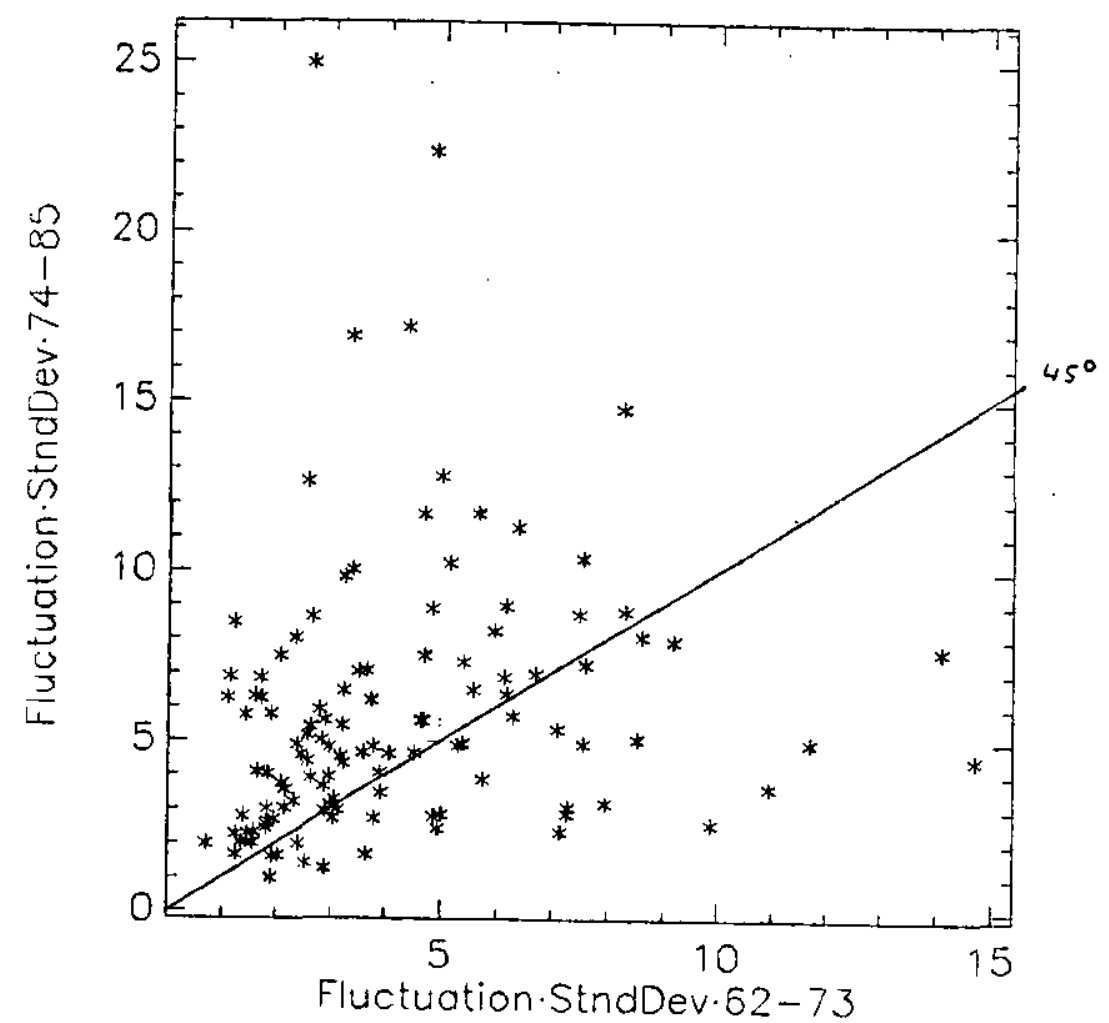


These graphs give a number of important messages. First, the data show instability in the underlying long-run growth patterns within each country; thus, assuming stable growth paths for each country and then studying their crosssectional variation gives results that are difficult to interpret. Second, the increasing fluctuation variability suggests that important disturbances-demand or productivity - are ongoing; a picture of the different economies as being largely perturbed only by World War II, and thereafter having been adjusting towards steady state equilibrium seems seriously flawed. Third, these graphs bear on the validity of conditional convergence, as studied in Barro and Sala-j-Martin [1991, 1992]. Fitting separate time trends for each country as I have done above can be viewed as extreme successful conditioning in a cross-country growth regression, namely, one where the explanatory variables yields an $R^{2}$ of 1 in explaining the underlying growth trends. Yet, even then, each country's income shows rising, rather than falling, variability over time. Figure 2 shows that conditional convergenceeither of the $\beta$ - or $\sigma$-variety in Barro and Sala-i-Martin's terminology-does not occur: shocks to each economy appear more important at the end of the sample than at the beginning. ${ }^{4}$

like "broken trends" and "unit roots" in time series data. While these graphs cannot be a satisfactory replacement for doing the analysis right, I go no further here with formal statistical methods: first, these calculations are intended to be only illustrative; and second, correctly bringing cross-country information to bear on the battery of known time-series tests for broken trends and unit roots is an entire research project in itself.

4 An alternative criticism of the convergence interpretations in Barro and Salai-Martin, similarly mindful of the nature of these ongoing shocks, is given in Den Haan [1992]. His analysis relies more on the subtlety that is called for in interpreting econometric evidence under the cross-equation restrictions implied by rational expectations economies. 

To keep within its space allotment, this paper addresses only the simplest such question: what are the long run tendencies of incomes and growth rates across countries?

I took each country's per capita income relative to world average as my basic data. Operator $M$ of $(t)$ is approximated by discretizing the set of possible values of relative incomes into intervals at $1 / 4,1 / 2,1$, and 2 . I also carry out the analysis for annual relative income growth rates; here I partition the set of possible values into intervals at $-4.5 \%,-1.5 \%, 1.5 \%$, and $4.5 \%$. (These choices-they seem a priori reasonable to me-turn out also to divide all the observed data points into roughly equal sized categories.) All relevant properties of $M$ are then described by a $5 \times 5$ Markov chain transition matrix whose $(j, k)$ entry is the probability that an economy in state $j$ transits to state $k$-in the following I will refer to this matrix and $M$ interchangeably. Low-numbered states correspond to low incomes or low growth rates. Thus, for example, state 1 in Table 1 below comprises per capita incomes no greater than one-fourth the world average; state 1 in Table 2 comprises annual growth rates no greater than $-4.5 \%$ relative to the world average.

The first panel of Table 1 contains the one-step annual transition matrix, estimated by averaging the observed one-year transitions over every year, from 1962-63 through 1984-85. The first column gives the total number of transitions with starting points in that income state. Thus, for example, the second row shows that over the entire sample-across 118 countries and across 23 years-643 observations fell in state 2, i.e., had incomes between one-fourth and one-half the world average. Of these, $92 \%$ remained in that same state in the following year.

Over this one-year horizon the predominant feature is-not surprisinglyhigh persistence; all diagonal entries exceed $90 \%$; other entries are nonzero only for the first state off the main diagonal. From state 2, a representative economy is (marginally) more likely to fall behind than to go ahead; similarly for state 4-this despite each higher cell's covering an ever larger-range.

The second panel of Table 1 is like the first, but now it describes the one 23year transition from 1962 through 1985; here, again, we see persistence, although less pronounced. For example, $7 \%$ of the economies originally at income levels between one-fourth and one-half of the world average (state 2) transited to incomes at world average and higher (states 4 and 5), over this longer horizon. This 
Table 1: Real GDP Per Capita (Relative to World Average) First order, time-stationary (1962 to 1984)

Grid: $(0,1 / 4,1 / 2,1,2, \infty)$; States: 5

\begin{tabular}{|l|ccccc|}
\hline & \multicolumn{5}{|c|}{ Upper Endpoint: } \\
\hline & $1 / 4$ & $1 / 2$ & 1 & 2 & $\infty$ \\
\hline$(456)$ & 0.97 & 0.03 & 0.00 & 0.00 & 0.00 \\
$(643)$ & 0.05 & 0.92 & 0.04 & 0.00 & 0.00 \\
$(639)$ & 0.00 & 0.04 & 0.92 & 0.04 & 0.00 \\
$(468)$ & 0.00 & 0.00 & 0.04 & 0.94 & 0.02 \\
$(508)$ & 0.00 & 0.00 & 0.00 & 0.01 & 0.99 \\
\hline Ergodic & 0.24 & 0.18 & 0.16 & 0.16 & 0.27 \\
\hline & \multicolumn{5}{|c}{ From second order specification: } \\
\hline Ergodic & 0.28 & 0.15 & 0.12 & 0.15 & 0.30 \\
\hline \hline
\end{tabular}

23-year transition (1962-1985)

\begin{tabular}{|c|c|c|c|c|c|}
\hline \multirow[b]{2}{*}{ (Number) } & \multicolumn{5}{|c|}{ Upper Endpoint: } \\
\hline & $1 / 4$ & $1 / 2$ & 1 & 2 & $\infty$ \\
\hline (17) & 0.76 & 0.12 & 0.12 & 0.00 & 0.00 \\
\hline (29) & 0.52 & 0.31 & 0.10 & 0.07 & 0.00 \\
\hline$(35)$ & 0.09 & 0.20 & 0.46 & 0.26 & 0.00 \\
\hline (17) & 0.00 & 0.00 & 0.24 & 0.53 & 0.24 \\
\hline$(20)$ & 0.00 & 0.00 & 0.00 & 0.05 & 0.95 \\
\hline \multirow[t]{2}{*}{ Ergodic } & 0.16 & 0.05 & 0.10 & 0.12 & 0.57 \\
\hline & \multicolumn{5}{|c|}{ From 2nd order spec. (22-year transition): } \\
\hline \multirow[t]{4}{*}{ Ergodic } & 0.20 & 0.09 & 0.13 & 0.12 & 0.47 \\
\hline & \multicolumn{5}{|c|}{ Stationary Estimate, iterated 23 times } \\
\hline & \multirow{2}{*}{\multicolumn{5}{|c|}{ 1/4 Upper Endpoint: }} \\
\hline & & & & & $\infty$ \\
\hline $1 / 4$ & 0.61 & 0.27 & 0.09 & 0.03 & 0.00 \\
\hline $1 / 2$ & 0.37 & 0.32 & 0.20 & 0.09 & 0.02 \\
\hline 1 & 0.14 & 0.23 & 0.31 & 0.25 & 0.07 \\
\hline 2 & 0.04 & 0.11 & 0.25 & 0.39 & 0.22 \\
\hline 5 & 0.00 & 0.01 & 0.04 & 0.12 & 0.82 \\
\hline
\end{tabular}


Table 2, Growth: Real GDP Per Capita (relative to World Average) First order, time-stationary (1962 to 1984)

Grid: $(-\infty,-4.5,-1.5,1.5,4.5, \infty)$; States: 5

\begin{tabular}{|l|ccccc|}
\hline \hline & \multicolumn{5}{|c|}{ Upper Endpoint: } \\
\hline$($ Number $)$ & -4.5 & -1.5 & 1.5 & 4.5 & $\infty$ \\
\hline$(463)$ & 0.34 & 0.22 & 0.17 & 0.13 & 0.14 \\
$(791)$ & 0.19 & 0.24 & 0.29 & 0.15 & 0.12 \\
$(501)$ & 0.12 & 0.18 & 0.40 & 0.18 & 0.12 \\
$(475)$ & 0.09 & 0.15 & 0.35 & 0.24 & 0.18 \\
\hline Ergodic & 0.17 & 0.11 & 0.19 & 0.20 & 0.33 \\
\hline & 0.18 & 0.18 & 0.29 & 0.18 & 0.17 \\
\hline Ergodic & & From second order specification: \\
\hline \hline
\end{tabular}

23-year transition (1962-1985)

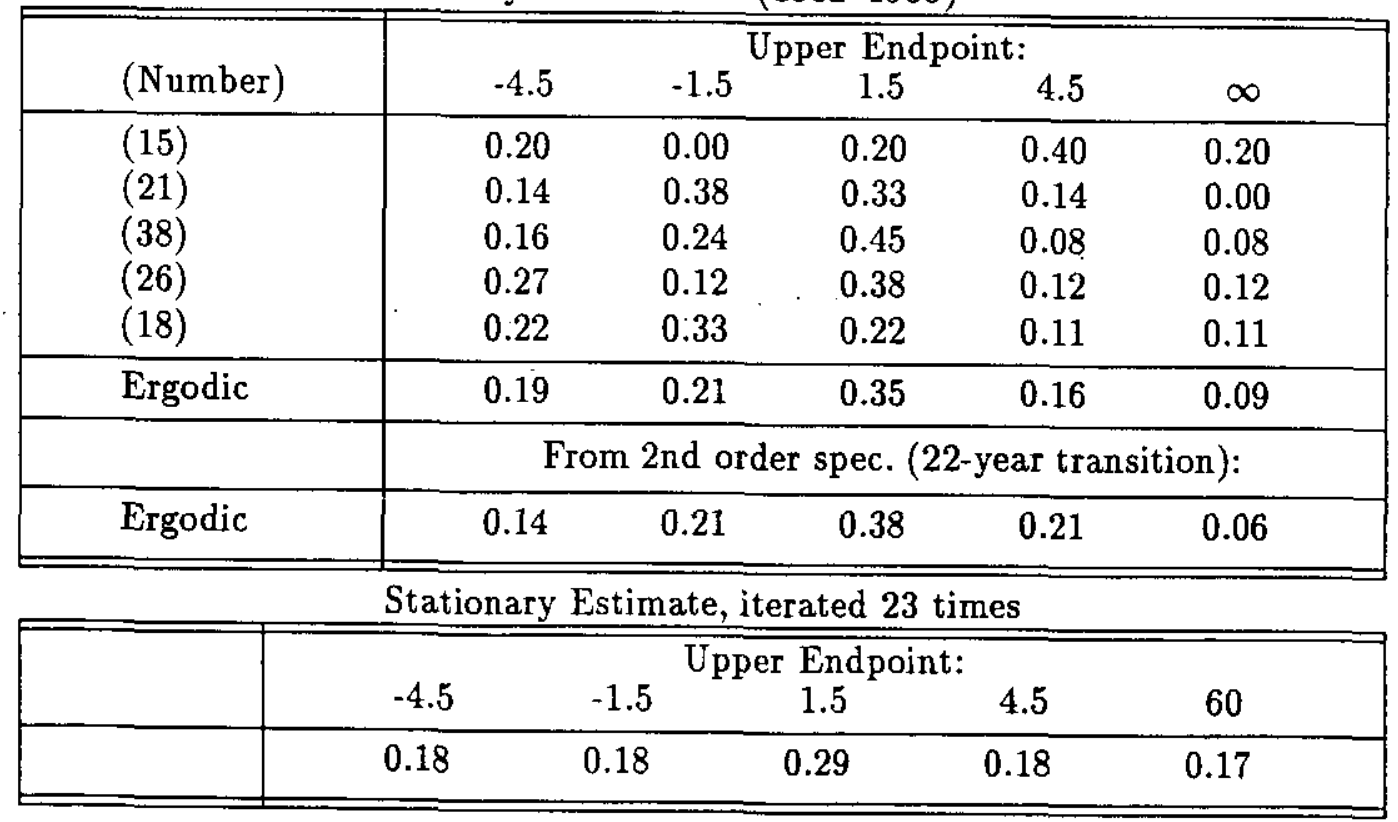


mobility is not all favorable though: of those same economies originally in state 2 , over one-half dropped to even poorer incomes. Looking down a neighborhood of the main diagonal suggests that at low income levels the greater tendency is to become even poorer, although some possibility for upward mobility always remains. At higher incomes, upward and downward mobilities just about balance; the highest income states, however, appear persistent-the $95 \%$ probability of the richest remaining richest is by far the largest entry in this transition matrix.

This informal description suggests cross-country incomes tending towards extremes at both high and low endpoints. We can make this precise by considering the ergodic distributions implied by these transition functions $M .^{5}$ These distributions are given below the corresponding transition matrices. For both of the first two panels of Table $1-M$ estimated by averaging annual transitions across time and over the long horizon-the implied ergodic distributions show first, a thinning in the middle, and second, an accumulation in both low and high tails. The two distributions show a higher probability at the upper tail-especially so for $M$ estimated over the long horizon. ${ }^{6}$

Recall that world per-capita income-the normalizing quantity-is a weighted

5 Nothing in the calculations enforces existence or uniqueness of an ergodic distribution-that precisely one such distribution was found is a consequence of the data. We should also emphasize that these steady-state distributions should not be read as forecasts of what will happen in the future-government policies might change; important, unforeseen events might occur. Rather, these distributions should be interpreted simply as characterizations of tendencies in the post-War history that actually realized.

6 One initially suspects this is due to (a) the way the cells are defined, the higher income cells covering a larger range, and (b) ongoing world-wide growth. Two points are worth noting: first, in the actual observed distributions-both in 1962 and over 1962-1984-the modal points occur, not in the larg-cover highincome states, but instead in the middle state (incomes between $50 \%$ and $100 \%$ of the world average). See the first column of each panel. Second, these distributions are for incomes relative to world average-world-wide growth is already absorbed in the normalization. 
average of each country's per capita income, with high weights assigned to the heavily-populated countries. Since incomes are non-negative, an accumulation at the high end of the distribution means that most of those countries getting richer have small populations. This behavior at the upper tail-convergence towards the richest countries, conditional on an economy being small and already relatively wealthy-should not distract from the distribution's behavior at the low end. Despite the cell here being the smallest it shows a similar accumulation in the ergodic distribution: countries caught in a low-income development trap remain there.

Each of the first two panels in Table 1 also report a second ergodic distribution. To examine robustness of the tendency of incomes towards the two extremes, I experimented with Markov chain transition models having dynamics beyond the first order. Formal statistical tests-of the Anderson-Goodman kind-suggest that the first-order model might be inadequate. Nevertheless, the implied ergodic distributions from richer structures almost invariably carry the same message: thinning out of the middle-income economies in favor of the very rich and very poor. The second ergodic distribution given in these panels is generated from a second order Markov chain (not presented here), estimated again by averaging across annual transitions and over the long horizon. The bimodal property of the ergodic distribution remains; the tendency for a poverty pile-up is now even more pronounced.

To check consistency of the short- and long-run first-order models, I iterated the one-period transition function of the short-run model to cover the same time span as that of the long-run one. The third panel of Table 1 reports this iterated one-step transition. Comparing the diagonal entries here with those for the second panel shows consistent under-prediction (except for state 2): the data show greater long-run persistence than predicted by the best-fitting first-order model. Also, every entry in the iterated transition function, except the two extreme endpoints, is strictly positive. By contrast, the long run model shows no transitions between very low- and very high-income states-nine entries in the opposing off-diagonal corners are all zero in the second panel (up to two decimal points). Again, this indicates higher persistence-lower cross-sectional mobility-in the data over long 
horizons than predicted by a time-homogeneous first order model. ${ }^{7}$

Table 2 presents exactly the same calculations as in Table 1, but now for growth rates relative to world average. First-order and second-order estimates imply quick convergence to the ergodic distribution; the ergodic distribution appears unimodal at and symmetric about zero. Although stochastic and time-varying, growth rates seem to display little pronounced, persistent movements. There is great cross-section mobility in growth rates-the first panel indicates that every state was reached by every other in one-step with some regularity: the minimum entry here is almost $10 \%$. Three of the five rows in the first panel have their largest entries off the diagonal. In the second panel, the largest entries in four of the five rows are off the diagonal. Persistently maintaining a very high (or very low) growth rate over short or long horizons is atypical.
\end{abstract}

7 The diagonal under-prediction is well-known from empirical Markov chain transition studies in sociology and occupational dynamics; see for instance Spilerman [1974] and Singer and Spilerman [1976]. One proposed solution to the inadequacy of the time-homogeneous first-order Markov model is a mover-stayer structure; elsewhere (Quah [1992]), I have considered long-run dynamic versions of such structures, allowing interesting patterns of time-heterogeneity and state dependence. That paper also explores more carefully some of the informal ideas that have been articulated in the text above. 


\section{Conclusions and Extensions}

Numerous studies on the determinants and dynamics of growth-of which I have cited only the most prominent-have investigated the empirical evidence in a way that, I argue here, obscures certain interesting and important features of the data. In this paper I have provided an alternative framework for studying the long-run dynamics of a rich panel of cross-country incomes. Despite its rough, first-pass nature, this study has provided interesting, robust characterizations of the tendency towards a two-camp world, divided between haves and have-nots, where escaping from the poverty trap is a low-probability proposition, either over the short- or long-run. In levels, there is greater persistence, at long horizons, than predicted by the best-fitting low-order time-homogeneous models; growth rates show high cross-section mobility and little own-persistence.

To refine these statements, to place more interpretable structure on the dynamics, and to bring in conditioning information (explanatory variables) all appear to be useful and feasible research projects. From a theoretical perspective, the descriptions here suggest that cross-country economic growth might be insightfully studied using models of income distributions--where the distributions are across, rather than within, entire economies. These would be usefully contrasted with the lessons from more traditional representative agent growth models.

From an econometric perspective, the more obvious statistical inadequacies here suggest fertile ground for further research-broadly viewed, this work concerns data where time-series and cross-sectional dimensions have the same order of magnitude. In probability theory, such data structures are known as random fields; econometric modelling of the dynamic- and cross-correlation properties of such structures is relatively unexplored. More narrowly, the transition functions above can be enriched by explicit time- and state-heterogenous modelling; their mobility implications can also be studied using measures such as that in Geweke, Marshall, and Zarkin [1986]. The arbitrary discretizing grid used to construct the Markov chain transition matrix is simply a crude nonparametric estimator for a distribution; sharper methods might yield correspondingly sharper insights. Also of interest is tracing out the intermediate-rather than just the one-period or alternatively steady-state-dynamics of these evolving distributions. 
These questions are all currently under investigation.

\section{References}

[1] Barro, Robert J. (1991), "Economic Growth in a Cross-Section of Countries", Quarterly Journal of Economics, 106(2):407-443, May.

[2] Barro, Robert J. and Sala-i-Martin, Xavier. (1991), "Convergence Across States and Regions", Brookings Papers on Economic Activity, (1):107-182, April.

[3] Barro, Robert J. and Sala-i-Martin, Xavier. (1992), "Convergence", Journal of Political Economy, 100(2):223-251, April.

[4] Den Haan, Wouter J. (1992), "Convergence in Stochastic Models", Working paper, Economics Dept., UCSD, July.

[5] Geweke, John, Marshall, Robert C., and Zarkin, Gary A. (1986), "Mobility Indices in Continuous Time Markov Chains", Econometrica, 54(6):1407-1423, November.

[6] Quah, Danny. (1992), "International Patterns of Growth: II. Persistence, Path Dependence, and Sustained Take-off in Growth Transition", Working paper, Economics Dept., LSE, September. (first draft July 1990).

[7] Singer, Burton and Spilerman, Seymour. (1976), "Sorne Methodological Issues in the Analysis of Longitudinal Surveys", Annals of Economic and Social Measurement, 5:447 -474.

[8] Spilerman, Seymour. (1974), "Extensions of the Mover-Stayer Model", American Journal of Sociology, 78(3):599-626.

[9] Summers, Robert and Heston, Alan. (1991), "The PennWorld Table (Mark 5): An Expanded Set of International Comparisons, 1950-1988", Quarterly Journal of Economics, 106(2):327-368, May. 


\section{Data Appendix}

The data are derived from that given in Summers and Heston (1991). Real per capita income is taken to from RGDPL (Laspeyres index); population is POP. Countries in the sample were selected by first disallowing those not having continuously available data on these two variables for the period 1960-1985. I then also excluded Kuwait-a 3-dimensional graph of the variables easily shows the Kuwait observation to dominate every other feature of the data. The remaining 118 countries are listed below (integers immediately before the country names are the indexes in the Summers-Heston database):

\begin{tabular}{rrlrrl}
\hline 1 & $(1)$ & Algeria & 2 & $(2)$ & Angola \\
3 & $(3)$ & Benin & 4 & $(4)$ & Botswana \\
5 & $(6)$ & Burundi & 6 & $(7)$ & Cameroon \\
7 & $(8)$ & CapeVerdeIs & 8 & $(9)$ & CentralAfrR \\
9 & $(10)$ & Chad & 10 & $(12)$ & Congo \\
11 & $(13)$ & Egypt & 12 & $(14)$ & Ethiopia \\
13 & $(15)$ & Gabon & 14 & $(16)$ & Gambia \\
15 & $(17)$ & Ghana & 16 & $(18)$ & Guinea \\
17 & $(19)$ & GuineaBiss & 18 & $(20)$ & IvoryCoast \\
19 & $(21)$ & Kenya & 20 & $(22)$ & Lesotho \\
21 & $(23)$ & Liberia & 22 & $(24)$ & Madagascar \\
23 & $(25)$ & Malawi & 24 & $(26)$ & Mali \\
25 & $(27)$ & Mauritania & 26 & $(28)$ & Mauritius \\
27 & $(29)$ & Morocco & 28 & $(30)$ & Mozambique \\
29 & $(31)$ & Niger & 30 & $(32)$ & Nigeria \\
31 & $(33)$ & Rwanda & 32 & $(34)$ & Senegal \\
33 & $(36)$ & SierraLeone & 34 & $(37)$ & Somalia \\
35 & $(38)$ & SouthAfrica & 36 & $(39)$ & Sudan \\
37 & $(40)$ & Swaziland & 38 & $(41)$ & Tanzania \\
39 & $(42)$ & Togo & 40 & $(43)$ & Tunisia \\
41 & $(44)$ & Uganda & 42 & $(45)$ & Zaire \\
43 & $(46)$ & Zambia & 44 & $(47)$ & Zimbabwe \\
45 & $(49)$ & Barbados & 46 & $(50)$ & Canada
\end{tabular}




\begin{tabular}{|c|c|c|c|c|c|}
\hline 47 & $(51)$ & CostaRica & 48 & (53) & Dominican Rep \\
\hline 49 & (54) & ElSalvador & 50 & (56) & Guatemala \\
\hline 51 & $(57)$ & Haiti & 52 & $(58)$ & Honduras \\
\hline 53 & (59) & Jamaica & 54 & $(60)$ & Mexico \\
\hline 55 & (61) & Nicaragua & 56 & $(62)$ & Panama \\
\hline 57 & (65) & TrinidadTobag & 58 & (66) & USA \\
\hline 59 & (67) & Argentina & 60 & (68) & Bolivia \\
\hline 61 & (69) & Brazil & 62 & $(70)$ & Chile \\
\hline 63 & (71) & Colombia & 64 & $(72)$ & Ecuador \\
\hline 65 & $(73)$ & Guyana & 66 & (74) & Paraguay \\
\hline 67 & (75) & Peru & 68 & $(76)$ & Suriname \\
\hline 69 & $(77)$ & Uruguay & 70 & $(78)$ & Venezuela \\
\hline 71 & (79) & Afghanistan & 72 & $(81)$ & Bangladesh \\
\hline 73 & $(82)$ & BurmaMyanmar & 74 & $(83)$ & China \\
\hline 75 & (84) & HongKong & 76 & (85) & India \\
\hline 77 & (87) & Iran & 78 & (88) & Iraq \\
\hline 79 & $(89)$ & Israel & 80 & $(90)$ & Japan \\
\hline 81 & (91) & Jordan & 82 & (92) & KoreaSouthR \\
\hline 83 & (94) & Malaysia & 84 & $(95)$ & Nepal \\
\hline 85 & (97) & Pakistan & 86 & $(98)$ & Philippines \\
\hline 87 & (99) & SaudiArabia & 88 & $(100)$ & Singapore \\
\hline 89 & $(101)$ & SriLanka & 90 & $(102)$ & Syria \\
\hline 91 & (103) & Taiwan & .92 & (104) & Thailand \\
\hline 93 & (107) & Austria & 94 & $(108)$ & Belgium \\
\hline 95 & $(109)$ & Cyprus & 96 & $(110)$ & Denmark \\
\hline 97 & (111) & Finland & 98 & (112) & France \\
\hline 99 & $(113)$ & GermanyWest & 100 & (114) & Greece \\
\hline 101 & (116) & Iceland & 102 & (117) & Ireland \\
\hline 103 & (118) & Italy & 104 & (119) & Luxembourg \\
\hline 105 & $(120)$ & Malta & 106 & (121) & Netherlands \\
\hline 107 & $(122)$ & Norway & 108 & (124) & Portugal \\
\hline 109 & (125) & Spain & 110 & $(126)$ & Sweden \\
\hline 111 & $(127)$ & Switzerland & 112 & (128) & Turkey \\
\hline
\end{tabular}




\begin{tabular}{llllll}
113 & $(129)$ & UK & 114 & $(130)$ & Yugoslavia \\
115 & $(131)$ & Australia & 116 & $(132)$ & Fiji \\
117 & $(133)$ & NewZealand & 118 & $(134)$ & PapuaNGuinea \\
\hline
\end{tabular}

All calculations and graphs were done using the author's program tsrf, a shell written in $\mathrm{C}$ and designed for work with random fields. 